\title{
Baccalaureate nursing students' confidence on patient safety
}

\author{
June M. Raymond ${ }^{* 1}$, Jennifer M. Medves ${ }^{2}$, Christina M. Godfrey ${ }^{2}$ \\ ${ }^{1}$ Cambrian College, Sudbury, Ontario, Canada \\ ${ }^{2}$ Queen's University, Kingston, Ontario, Canada
}

Received: November 21, 2016

DOI: $10.5430 /$ jnep.v7n6p56
Accepted: January 6, 2017

Online Published: January 17, 2017

\begin{abstract}
High rates of patient safety events led to media attention and litigation that sparked the patient safety movement. Gaining awareness of nursing students' confidence levels on patient safety will reveal their perceptions of their competency. A quantitative descriptive cross-sectional method was employed. The Health Professional Education in Patient Safety Survey was used to determine how 458 baccalaureate nursing students from two nursing programs in Ontario, Canada rate their confidence levels on patient safety. Nursing students are the most confident on Clinical Safety topics and least confident on topics related to Human and Environmental Factors and Culture of Safety. Overall, they are more confident in the classroom than in the clinical settings with fourth year students expressing lower degrees of confidence on many of the areas. Education programs need to reinforce patient safety and educate students on their responsibilities to protect patients/clients. Programs need to target strategies to reduce student fears, promote error reporting, and system improvements.
\end{abstract}

Key Words: Patient safety, Nursing student, Confidence, Education

\section{INTRODUCTION}

Patient safety has become a global concern within the healthcare system due to the high occurrence of patient safety events and near misses across all sectors of care. ${ }^{[1-7]}$ As defined by the Canadian Patient Safety Institute (CPSI), patient safety is "the pursuit of the reduction and mitigation of unsafe acts within the healthcare system, as well as the use of best practices shown to lead to optimal patient outcomes". ${ }^{6]}$ In recognition of high error rates the Institute of Medicine (IOM) mandated that there be improvements to health education programs by increasing the amount of content related to safety. ${ }^{[8]}$ Incorporating changes to health education follows the assumption that education is a viable strategy for improving the quality of patient care. While education is the foundation for knowledge, understanding the way that knowledge transitions into practice is of the upmost importance.

Although all health care providers are responsible for providing safe care, nurses are the largest group of healthcare providers in direct contact with patients and have the greatest potential to affect the quality of care ${ }^{[9-11]}$ Historically, nursing has always been a patient focused profession that has placed great emphasis on patient care and outcomes. ${ }^{[12]}$ The nursing regulatory body in Ontario is the College of Nurses of Ontario (CNO) and it has developed professional standards that encompass entry-to-practice competencies to ensure that nurses that enter the practice domain are competent to provide safe care. ${ }^{[12,13]}$ Registered Nurses (RNs) in

\footnotetext{
*Correspondence: June M. Raymond; Email: June.raymond@cambriancollege.ca; Address: Cambrian College, 1400 Barrydowne Road, Sudbury,
} Ontario, P3A 3V8, Canada. 
Ontario have undertaken a four-year university baccalaureate degree, are accountable for their actions, and are required to provide care in a safe manner. ${ }^{[13]}$

The IOM in the United States (US) mandated changes to health education however it is unclear the degree to which this has happened. Globally, there are few studies that have explored patient safety integration within educational curricula. In the United Kingdom, Japan, and United States, researchers have found little to no patient safety integration within nursing curricula and found little to no patient safety integration within the educational materials. ${ }^{[14-19]}$ This does not necessarily mean that the content is absent from these programs but rather that there is very minimal documentation of its existence. There is no published evidence on the state of integration of patient safety topics within Canadian nursing curricula. While enhancing educational curricula is important, it does not necessarily translate into student knowledge and confidence at the bedside. Although there is no evidence to substantiate the quantity of patient safety within nursing education in Canada the premise is that there is some degree of integration due to the patient safety movement and the previously stated mandate from the IOM. Integration of knowledge into practice or knowledge translation is a lengthy process and often takes one or two decades before research knowledge is actively incorporated into practice, a phenomenon referred to as the theory to practice gap. ${ }^{[20]}$ Knowledge translation and bridging the theory to practice gap is vital to improving patient outcomes, the health care system, and the practice of health care providers.

Gaining an understanding of nursing students' perspectives on their confidence levels of patient safety topics will help to provide a more accurate representation of their perception of their practice in the clinical setting. It could also provide some insight into the extent of the current 'theory to practice gap' in patient safety. Furthermore, it can identify areas where additional educational emphasis and training are needed

The literature that explores the topic of nursing student confidence about patient safety is limited. The purpose of this study was to describe baccalaureate nursing students' perspectives of their confidence levels on various patient safety topics. Students from two university settings in Ontario, Canada were included in this study. Baccalaureate nursing students were recruited to gain an understanding of what the current nursing student populations' confidence levels are on patient safety topics. The research question that guided this study was: How confident are baccalaureate nursing students in what they are learning about patient safety within their nursing education?

\section{Objective}

The purpose of this study was to explore nursing students' perspectives on their levels of confidence related to safety topics. Scant research describes students' safety confidence. Nursing students have high rates of direct patient contact and students need to gain confidence to practice safely. This research can provide valuable insight that can assist nursing education in developing educational initiatives that target this area.

\section{Methods}

\subsection{Sample}

A quantitative cross-sectional descriptive study was conducted with students from all four years of the Bachelor of Science in Nursing programs from two university settings in Ontario, Canada during the 2014/15 academic year. All students had to be enrolled in one of the identified programs, have access to a computer and the Internet. All students indicated that they had participated in a minimum of one clinical placement.

\subsection{Study instrument}

The data was collected using an adapted web based version of the Health Professional Education in Patient Safety Survey (H-PEPSS), a tool originally created to ask recent health practitioner graduates for their perspectives on broad areas of patient safety competencies. ${ }^{[20]}$ Duhn and colleagues (2012) adapted the original survey tool to use with nursing students. ${ }^{[21]}$ The original tool was developed following the Canadian Patient Safety Framework (CPSF) ${ }^{[6]}$ and has three sections with 38 items in total. The first section has 27 questions assessing seven patient safety subscales (Clinical Safety, Working in Teams, Communicating Effectively, Managing Safety Risks, Human and Environmental Factors, Adverse Events, and Culture of Safety). The students were asked to rate their confidence separately for both classroom and clinical settings using a five point Likert scale ( $1=$ strongly disagree, $2=$ disagree, $3=$ neutral, $4=$ agree, $5=$ strongly agree, and a 'don't know' option). The second section of the tool has seven questions about broader patient safety issues in their education and students were asked to rate their answers using the same five point Likert scale as above except without the 'don't know' option. The third section has four questions asking students to rate their comfort levels speaking up about patient safety using the five point Likert scale, again the 'don't know' response was not an option. The original H-PEPSS was validated with a sample of interprofessional new healthcare graduates using a Confirmatory Factor Analysis (CFA) and revealed an internal consistency reliability of 0.80 or greater for all six factors and the version adapted for use in students was validated with medical 
students where a good model of fit was attained using acceptable indices of fit (Confirmatory Fit Index $>0.95$, Root Mean Square Error of Approximation < 0.06). ${ }^{[22]}$ Approval to use the H-PEPSS in this research study was received from its developers. Demographic characteristics, including age, gender, year in the program, and educational institution were also collected.

\subsection{Ethics and data collection procedure}

After the ethics review board approvals were obtained from each institution, an administrative member from each nursing department was sought to assist with recruitment of participants. On behalf of the researchers, the administrative staff member from each institution forwarded an email invitation to all students enrolled in their baccalaureate nursing program. This email invitation included a description of the study with a web link to the survey. The questionnaire was administered electronically via FluidSurvey ${ }^{T M}$ and was open to participants for a 15 consecutive week period during the fall and winter terms of the 2014/15 academic year for both sites. The students who accessed the link and completed the survey were deemed to have provided consent to participate. To ensure participant anonymity and confidentiality unique identification codes were assigned to each participant questionnaire. Two reminder emails were forwarded to all students at two-week intervals. The researcher also arranged a date and time to meet students at each institution or through virtual means to present a brief outline of the study to any students who had any questions. There were small numbers of students (less than 5) who opted to participate at each site and there were minimal questions asked by students. The administrative staff reported that 1,400 students were eligible in total and invited to participate (University A-600 participants; University B-800 participants). Four hundred and fifty-eight responses were received.

\subsection{Analysis}

Data was analyzed using IBM SPSS Statistics for Macintosh, Version 22.0 C . The questions for each of the patient safety subscales were averaged to create seven subscales with a score ranging from 1 to 5 . Univariate descriptive statistics for each of the seven subscales in both the classroom and clinical settings were calculated. Percentages were calculated and are presented for categorical data. Means and standard deviations are presented for continuous data. Pearson's correlation tests were calculated to determine if there were relationships between classroom and clinical perceptions in patient safety subscales. A Pearson's Correlation coefficient in the 0 to 0.2 range is considered very weak, a 0.2 to 0.4 coefficient signifies a weak relationship, a 0.4 to 0.6 indicates a moderate strength, and 0.6 to 0.8 translates to a strong relation, and lastly a very strong relationship would reveal a 0.8 to 1.0 correlation coefficient. To determine practical or educational significance/relevance raw effect sizes were calculated. ${ }^{[23]}$ In education research, an effect size of greater than 0.5 is seen as having a medium effect and thus educationally significant. ${ }^{[24]}$ To test for statistically significant differences across academic years in each of the seven subscales unpaired oneway analysis of variances and Tukey's post-hoc tests were conducted.

\section{RESUltS}

\subsection{Demographic details}

Ninety-four percent $(n=432 / 459)$ of the participants were female. Eighty-eight percent of participants were 27 years of age or younger (see Table 1).

Table 1. Demographic data of participants $(n=458)$

\begin{tabular}{ll}
\hline Characteristic & $\mathbf{n}(\mathbf{\%})$ \\
\hline Gender & $432(94)$ \\
Female & $26(6)$ \\
Male & \\
Age & $160(35)$ \\
$18-22$ & $244(53)$ \\
$23-27$ & $50(20)$ \\
$28-32$ & $4(2)$ \\
$33-40$ & \\
Institution & $208(45)$ \\
University A & $250(55)$ \\
University B & \\
Response Rate & $34.7 \%$ \\
University A & $31.3 \%$ \\
University B & \\
Year in Program & $138(30)$ \\
Year 1 & $102(22)$ \\
Year 2 & $88(19)$ \\
Year 3 & $130(29)$ \\
Year 4 &
\end{tabular}

\section{Patient safety topic content area (Section One of $\mathrm{H}$ - PEPSS)}

The means and standard deviations for each of the seven subscales for both the classroom and clinical settings are presented in Table 2. The students expressed the highest level of confidence (classroom mean of 4.7 , clinical mean of 4.4) on the topics of hand hygiene, infection control, and medication practices, which were subtopics included in the Clinical Safety subscale. In the classroom setting, the students were the least confident (mean of 4.3 on the 5-point scale) on topics related to the Working in Teams, Human and Environmental Factors, Adverse Events, and Culture of Safety, however, this rate is still within the agreement rating of the scale so the majority of students did feel confident. In 
the clinical setting, the students were least confident (mean of 4.0 on the 5-point scale) on topics related to Working in Teams and the Culture of Safety such as the nature of systems, impact of having a supportive environment, and the complexity of the environment. It is also important to note that within all of the seven subscales, the students expressed more confidence in their knowledge within the classroom than clinical settings.

Since students are provided with education within the class- room and clinical settings the correlation between these learnings was assessed. Table 2 summarizes this data. For each of the seven subscales, a positive correlation was noted with the Pearson's Correlation coefficients in the 0.3 to 0.5 range, with 0.3 signifying weak strength relationships and 0.5 translating to a moderate strength relationship between classroom and clinical settings. Based on raw effect sizes, there are no educationally significant differences $(>0.5)$ found between the classroom and clinical means for any of the patient subscales (see Table 2).

Table 2. Relationship between perspectives of classroom and clinical learning on patient safety subscales

\begin{tabular}{lllllll}
\hline Subscale & $\begin{array}{l}\text { University A } \\
(\mathbf{n = 2 0 8 )}\end{array}$ & $\begin{array}{l}\text { University B } \\
(\mathbf{n = 2 5 0 )}\end{array}$ & $\begin{array}{l}\text { University A and } \\
\text { B Classroom } \\
\text { Mean (SD) }\end{array}$ & $\begin{array}{l}\text { University A } \\
\text { and B Clinical } \\
\text { Mean (SD) }\end{array}$ & $\begin{array}{l}\text { Pearson } \\
\boldsymbol{r}\end{array}$ & $\begin{array}{l}\text { Effect } \\
\text { Size }\end{array}$ \\
\hline Clinical Safety & $4.5(.4)$ & $4.6(.4)$ & $4.7(.5)$ & $4.4(.5)$ & .5 & .28 \\
Working in Teams & $4.2(.5)$ & $4.2(.5)$ & $4.3(.6)$ & $4.0(.6)$ & .4 & .24 \\
Communicate Effectively & $4.4(.4)$ & $4.4(.5)$ & $4.5(.5)$ & $4.2(.5)$ & .4 & .29 \\
Manage Safety Risks & $4.2(.5)$ & $4.3(.5)$ & $4.4(.6)$ & $4.2(.5)$ & .4 & .19 \\
Human and Environmental Factors & $4.1(.5)$ & $4.3(.5)$ & $4.3(.6)$ & $4.1(.5)$ & .3 & .18 \\
Adverse Events & $4.2(.4)$ & $4.3(.5)$ & $4.3(.6)$ & $4.1(.5)$ & .3 & .18 \\
Culture of Safety & $4.1(.4)$ & $4.2(.5)$ & $4.3(.5)$ & $4.0(.5)$ & .3 & .29 \\
\hline
\end{tabular}

$* p$ Value $<.01$ for all subscales.

In addition to assessing the relationship between classroom and clinical learning, the differences between perceptions in the academic years were explored. Table 3 includes the means by academic year for all the seven subscales for both classroom and clinical settings. Those that indicate a $p$ value of less than .05 were noted as statistically significant. Tukey's post hoc was conducted to discover where the differences were located and to allow effect sizes to be calculated to determine educational significance. Raw effect sizes revealed that there were no educationally significant differences between classroom and clinical means $(>0.5)$.

\subsection{Broader patient safety (Section Two of H-PEPSS)}

Dichotomized data was calculated (agree/strongly agree versus neutral/disagree/strongly disagree) of the students' perspectives on broader patient safety issues addressed within their educational program by academic years in the program. In summary, $83 \%$ to $94 \%$ of all students felt that their scope of practice was clear to them with year one students expressing the highest mean at 4.1 on a 5-point scale. Between $51 \%$ and $61 \%$ of students expressed that there is consistency demonstrated by preceptors in dealing with patient safety issues. In response to the question asking whether the students agree that they "have sufficient opportunity to learn and interact with interdisciplinary team members", only $74 \%$ of the fourth year students believed they did whereas $93 \%$ of third year students, $84 \%$ of second year students, and
$91 \%$ of first year students expressed that they have sufficient opportunities. High percentages of students (86\% to 97\%) perceived that they were gaining a solid understanding of reporting adverse events and near misses with the fourth year students again being the lowest response at $86 \%$. For the two questions related to overall patient safety integration in their program and clinical aspects of safety, between $94 \%$ and $100 \%$ of the students agreed that these concepts were well covered within their programs with the fourth year students expressing the least confidence in both of these questions at 94\%. The last question in this section was whether the students perceived that 'systems' aspects were well covered in their education and the agreement with this question ranged from $91 \%$ and $92 \%$ for first and second year students respectively, $84 \%$ of third years were in agreement and $78 \%$ of the fourth year students expressed agreement.

\subsection{Comfort speaking up (Section Three of H-PEPSS)}

The questions in this section of the survey are particularly interesting because students may have knowledge but are afraid to speak up on issues that could affect the safety of their patients. Seventy-four percent of first year students stated they could approach someone they see practicing unsafely compared to $69 \%$ of second years, $73 \%$ of third years, and $63 \%$ of fourth years. The second question was whether the students feared disciplinary actions when they make errors and $46 \%$ of first year students, $53 \%$ of second years, 
$55 \%$ of third years, and $68 \%$ of the fourth year students feared punitive repercussions. Sixty-five percent of fourth year students stated it is difficult to question actions by those in authoritative positions whereas $74 \%$ of first year students, $75 \%$ of second year students, and $80 \%$ of third years reported difficulty with this task. Between $55 \%$ and $70 \%$ of students agreed that the focus is on systems when an error is made rather than the individual most responsible with the fourth years expressing the lowest percentage of agreement.

Table 3. Students' perspectives of confidence in patient safety in classroom and clinical learning

\begin{tabular}{|c|c|c|c|c|c|c|}
\hline \multirow{2}{*}{ Subscale } & \multicolumn{6}{|c|}{ Means (SD) } \\
\hline & Year One & Year Two & Year Three & Year Four & ANOVA $F$ test & $p$ Value \\
\hline \multicolumn{7}{|c|}{ Clinical Safety } \\
\hline Classroom & $4.7(.5)$ & $4.6(.5)$ & $4.8(.4)$ & $4.6(.5)$ & 4.46 & .00 \\
\hline Clinical & $4.4(.5)$ & $4.4(.5)$ & $4.6(.5)$ & $4.4(.6)$ & 2.23 & .08 \\
\hline \multicolumn{7}{|c|}{ Working in Teams } \\
\hline Classroom & $4.4(.5)$ & $4.3(.5)$ & $4.4(.5)$ & $4.2(.6)$ & 2.83 & .38 \\
\hline Clinical & $4.1(.5)$ & $4.1(.6)$ & $4.1(.6)$ & $3.9(.6)$ & 7.10 & .00 \\
\hline \multicolumn{7}{|c|}{ Communicate Effectively } \\
\hline Classroom & $4.6(.5)$ & $4.5(.5)$ & $4.6(.5)$ & $4.5(.6)$ & 1.70 & .17 \\
\hline Clinical & $4.3(.5)$ & $4.2(.5)$ & $4.4(.5)$ & $4.1(.6)$ & 7.16 & .00 \\
\hline \multicolumn{7}{|c|}{ Managing Safety Risks } \\
\hline Classroom & $4.4(.5)$ & $4.4(.7)$ & $4.4(.6)$ & $4.3(.6)$ & 2.03 & .11 \\
\hline Clinical & $4.2(.4)$ & $4.2(.6)$ & $4.1(.5)$ & $4.0(.5)$ & 3.58 & .01 \\
\hline \multicolumn{7}{|c|}{ Human and Environmental Factors } \\
\hline Classroom & $4.4(.6)$ & $4.4(.6)$ & $4.4(.6)$ & $4.2(.6)$ & 3.57 & .01 \\
\hline Clinical & $4.1(.5)$ & $4.2(.5)$ & $4.0(.5)$ & $4.0(.6)$ & 1.95 & .12 \\
\hline \multicolumn{7}{|c|}{ Adverse Events } \\
\hline Classroom & $4.4(.5)$ & $4.3(.7)$ & $4.3(.6)$ & $4.2(.7)$ & 2.26 & .08 \\
\hline Clinical & $4.1(.4)$ & $4.1(.4)$ & $4.1(.5)$ & $4.0(.5)$ & 1.14 & .33 \\
\hline \multicolumn{7}{|c|}{ Culture of Safety } \\
\hline Classroom & $4.3(.5)$ & $4.3(.5)$ & $4.3(.6)$ & $4.2(.5)$ & 1.29 & .28 \\
\hline Clinical & $4.1(.4)$ & $4.1(.4)$ & $4.1(.4)$ & $3.8(.6)$ & 7.46 & .00 \\
\hline
\end{tabular}

\section{Discussion}

The students in the current research study expressed the highest confidence in the Clinical Safety subscale followed by the Communicating Effectively subscale within both classroom and clinical settings which are consistent with previous research on this topic. ${ }^{[16,20]}$ As well, positive correlations between the classroom and clinical learnings were noted. The first year students in the current study expressed high rates of confidence in the classroom (mean $=4.7$ ) second only to third years (classroom mean $=4.8$ ) which was not found in the previous literature. ${ }^{[21,25]}$ The current students expressed higher rates of confidence on all patient safety topics compared to the students in the previous studies. Consistently, the fourth year students express a lower confidence level than other years for multiple areas.

For all of the specific broader patient safety questions, the students in the current study expressed more agreement than the students in previous research by Lukewich and colleagues (2015) with the biggest difference in students' perceptions related to the statement asking if students agree that 'system aspects of patient safety are well covered in their program' with approximately $40 \%$ more students in the current study agreeing with the statement than in the previous study. ${ }^{[25]}$ These differences may suggest that the students that were included in the current study may be enrolled in programs where there have been curriculum changes that have emphasized systems issues and approaches.

Both the current and previous research exploring this topic had approximately $50 \%$ of students expressing that 'there is consistency in how patient safety issues are dealt with by preceptors in the clinical setting'. This suggests that consistency in preceptor actions continues to be an ongoing concern. Consistency has been identified as a strategy for enhancing student accountability and confidence in recognizing and reporting errors so the greater proportion of students who feel their preceptors act consistently, the greater likelihood students are practicing in a manner that is reflective of professionally accountable practice. This comparison sug- 
gests that preceptor consistency is a real issue across multiple programs in Ontario and is an area that requires further investigation. Greater proportions of students from the current study expressed that they feel comfortable to approach someone practicing unsafely than in the Lukewich et al.'s (2015) study. Speaking up is a requirement for nurses as per their Code of Ethics. ${ }^{[12]}$ Nurses are responsible for speaking up on their patient's behalf and protecting their patients from harm. ${ }^{[12]}$ This could suggest that students are becoming more confident in their questioning attitudes, which demonstrates professional practice growth and awareness of its importance in providing safe practice.

The sample of students in the current study reported less fear of disciplinary repercussions than the students in previous research, which could indicate that students are seeing the benefit and importance of reporting errors. It could also suggest that the educational environments are changing or perhaps that teachers are encouraging them to report errors and concerns and they are supporting students in this. As well, higher proportions of students in the current study reported that the focus of errors is on systems issues rather than individual issues compared to the students in the Lukewich et al., 2015 study. ${ }^{[25]}$ This could indicate that students are becoming more aware of the influence of systems on safe practice and perhaps recognizing the value of reporting from a safety perspective.

\subsection{Implications for education/practice}

Strategies to assist with knowledge transfer into the clinical setting need to be creative and student focused and perhaps even student driven. The students consistently ranked their confidence rates higher in the classroom settings, which suggests difficulty translating this knowledge to the practice setting. Although these differences were not found to be educationally significant this is a pattern that is worth noting. Creating more opportunities for reflection-in-action is a strategy that could be used to help facilitate this process. ${ }^{[26]}$ Creating stronger relationships between clinical and theory instructors could help to bridge this theory to practice gap and create a more consistent response to patient safety events, which was identified as a problem area across all years of the programs.

The fourth year students seemed to have lower levels of confidence across the majority of the topics within the survey. Although lower levels of confidence were expressed this does not mean that the fourth years are less competent than other years. Barnsley and colleagues (2004) found that there was no relationship between expressed self-confidence and observed competence in clinical skills although Sulosaari and colleagues (2012) stated that students' expressed confi-

Published by Sciedu Press dence is linked to their individual sense of competence. ${ }^{[27,28]}$ It would be interesting to explore the reasons why fourth year students have expressed lower levels of confidence as one would have expected the fourth year students to have higher confidence levels simply due to their position in the educational process and their soon to be graduate status, although this phenomenon has also been noted by other researchers. ${ }^{[20,25]}$

Nurses are responsible for the total care of their patients including advocating for them when the need arises. A higher degree of confidence is required to speak up on a patient's behalf. The low confidence rates of fourth year students and the findings that $63 \%$ to $65 \%$ of these students expressed they have difficulty speaking up/advocating on topics related to patient safety identifies an area that needs further improvement within nursing education. Incorporating a higher level of assertiveness training into the current curriculum could help improve students' confidence rates.

Fear of repercussions has been documented throughout the years as a major reason for low error reporting rates and this study found similar feelings of fear amongst students. ${ }^{\text {[29-31] }}$ Sixty-eight percent of fourth year students in this study expressed that they fear repercussions. It is imperative that we create and sustain a healthcare culture in which errors and harmful incidents as the end point of multiple factors are recognized (environmental, system, provider) and these events are seen as learning opportunities to improve our systems of care. This culture would support and encourage the reporting of errors by all healthcare providers. In particular, it would reduce the fears of the most inexperienced members of the healthcare teams - the students - modeling for them care that is safe and patient focused. Although this culture has been recognized within many studies, it is slow to transition to the clinical environment. Creating a reporting system that is not punitive and more constructive could help facilitate this transition. Emphasizing the errors as systems related would also help with improving students' confidence in a number of the areas that demonstrated a low percentage of student confidence particularly with fourth years $(55 \%)$.

Students place high value on consistency in preceptor behaviour. ${ }^{[32-35]}$ Since this was an issue that yielded concern it is important to address development of policies and procedures for dealing with issues. Processes need to be transparent and implemented on a consistent basis by classroom and clinical faculty/preceptors as consistency and effective communication are qualities of leadership that are highly valued. ${ }^{[32,36]}$ This may require more and/or stronger partnerships between full-time faculty and clinical preceptors. ${ }^{[37]}$ Students need to be knowledgeable about their education, 
system vulnerabilities, consequences of actions, and learning in all phases of their education and practice. ${ }^{[38]}$

Since patient care and patient safety events occur in clinical, it is imperative that initiatives are brought into the clinical placements that include the elements of patient safety and educators need to stress the importance of safety. With this research finding that students are more confident in the classroom than in the clinical settings, strategies that focus on enhancing knowledge, skills, and attitudes in the clinical setting would help to enhance students' confidence in these settings. Simulation has gained popularity and could provide students with opportunities to perform skills, make errors, and study errors without harming patients. ${ }^{[39]}$ This practice could build needed confidence and bridge the transition into the clinical setting. Employing learning opportunities through simulation that help students to develop thinking patterns that are rooted in early detection, recognition, and intervention would help to improve their self-confidence. ${ }^{[40]}$ Including a reporting system that can be employed when errors are made in simulation could help to increase student's familiarity with the reporting process. This familiarity could lead to increased confidence to report errors.

Emphasis on the system aspects of safety needs to be further integrated into curriculum and emphasized in both classroom and clinical settings. Incorporating this systems approach could also be an effective way of reducing individual fears when an error is made by enhancing student knowledge of the culture of safety, his/her responsibilities, vulnerabilities of systems, and the importance of safe practice. ${ }^{[38]}$ The emphasis on the systems approach to errors could be a further element in the suggestion above regarding including formal reporting during simulation. If reporting is incorporated in simulation, root cause analyses could be conducted by faculty and students collaboratively with a focus on what systems issues influenced the error. This would result in students gaining an increased understanding of systems issues and their relationship to errors.

Appropriate assertiveness training and an emphasis on the role of the nurse as patient advocate could be increased in student education. As nurses are required to advocate for their patients, it is necessary that they speak up on behalf of patients. Assertiveness training could help to provide them with the skillset to maximize their confidence and minimize their discomfort questioning others, which could potentially reduce the harm to patients. ${ }^{[41]}$ Training that assists students with developing professional identities could help to enhance students' comfort to speak up. ${ }^{[41]}$ Effective use of assertive communication is a difficult skill and one that could be included within communication or interpersonal relationship courses within nursing education.

Providing junior level nursing students with a senior level nursing peer mentor could be a strategy to help build confidence that would encourage both students to feel more confident and less fearful when making an error thereby making speaking up a more manageable task. ${ }^{[42,43]}$ Furthermore, ensuring that students are given ample opportunity to report, educated on the importance of it, and shifting to a culture of accountability can reduce some of the known fears that nursing students, and even practicing nurses, experience. ${ }^{[38]}$

Within the classroom setting, further educational initiatives that aim to reduce fears are needed. Nurse educators need to ensure that students are prepared for the environment by reinforcing the systems aspects of errors, as opposed to individual blaming, while stressing the importance of reporting errors to improving the flawed systems. Use of clinical simulation will help to build student skills and confidence, which could reduce students' fears. Providing students with checklists for assessments could help students to feel more prepared which could also reduce student fears. Having a clear and accessible policy on management of student errors will help to ensure transparency and reduce students' fears of unknown repercussions.

Within the clinical settings, some strategies to reduce students' fears of repercussions could include a blameless reporting system, debriefing following events, and using errors as teachable moments without identifying the student and as a group reflection. Nurse educators should employ constructive teaching methods to foster a positive learning environment such as through the use of anecdotes, stories, or having a warm attitude toward clinical content. ${ }^{[4]}$

\subsection{Study strengths and limitations}

The large sample size is a strength of this study despite the use of an online tool, which typically yields lower response rates than paper-based questionnaires. ${ }^{[45,46]}$ Although the sample size is large the response rate of $32.7 \%$ could suggest that the sample is not representative of the population. Since the researcher has no relationship with the students this would have decreased the social desirability bias but there is no way of knowing for certain. It is likely, however, that the students who participated in the study are those that are passionate about the topic being studied. It is also important to acknowledge that while obtaining insight into students' confidence on patient safety topics is valuable, it is only one measure and may not be indicative of their actual professional practice. The findings in this study are limited to the programs that were explored and to the participants that participated and may not be generalizable to the overall 
nursing student population in Ontario, Canada. Lastly, the study is cross-sectional in nature and has presented data at one point in time.

\section{Conclusions}

This study presented baccalaureate nursing students' perspectives of patient safety subtopics, broader patient safety topics, and comfort levels speaking up. It highlighted some areas that require further emphasis within the current education programs and revealed areas that are well translated to the students. Additional research is needed with students from nursing programs province wide to gain a clear picture of their perspectives of their confidence on patient safety topics and so that further comparisons can be done and generalizations can be made. Further research into factors that affect nursing students' comfort levels to speak up would help to elucidate the issue. Preceptor consistency or lack thereof in response to safety issues is an area that requires further investigation and emphasis within research and educational programs.

This study showed differences in the current students' confidence levels from those students studied within the research by Duhn and colleagues (2012) and Lukewich and colleagues (2015). The research by Duhn and colleagues (2012) and
Lukewich and colleagues (2015) were conducted in one nursing program whereas the current study collected data from two different nursing programs. It is unclear what factors could have contributed to differences in the baccalaureate student perceptions. It could be due to the increasing emphasis on patient safety in nursing programs and the increased use of patient safety terms and concepts within nursing education. Consistently, the fourth year students are less confident than students in other years which could be due to an increase in self-awareness. Students may be more aware of their limited knowledge as they near graduation and may be more cognizant of the reality that they are novice practitioners and have rated their confidence with this in mind. This premise is supported by Sears and colleagues (2014) who found that novice learners tend to be overconfident and advanced learners tend to underrate their confidence. ${ }^{[47]}$ The students rated their confidence on clinical safety topics, which includes the skill of medication administration, highly. Based on this research study, one could suggest that nursing students are becoming more confident on safety topics but it is too premature to make this declarative statement as it is a phenomenon that is underexplored and requires further investigation.

\section{Conflicts of InTEREST Disclosure}

The authors declare that there is no conflict of interest.

\section{REFERENCES}

[1] Baines RJ, Langelaan M, de Bruijne MC, et al. Changes in adverse events rates in hospitals over time: A longitudinal retrospective patient record review. BMJ Qual Saf. 2013 Apr; 22(4): 290-98. https://doi.org/10.1136/bmjqs-2012-001126

[2] Baker GR, Norton PG, Flintoft V, et al. The Canadian adverse events study: The incidence of adverse events among hospital patients in Canada. CMAJ. 2004 May; 170(11): 1678-86. https: //doi.org/10.1503/cmaj.1040498

[3] Blais R, Sears NA, Doran D, et al. Assessing adverse events among home care clients in three Canadian provinces using chart review. BMJ Qual Saf. 2013 Dec; 22(12): 989-97. https ://doi .org/10 $.1136 / \mathrm{bmjqs}-2013-002039$

[4] Canadian Patient Safety Institute. Safety at home: A PanCanadian home care safety study. 2013. Available from: http://www . patientsafetyinstitute.ca/English/resear ch/commissionedResearch/SafetyatHome/Documents/Saf ety $\% 20$ At $\% 2$ Home $\% 20$ Care.pdf

[5] Classen DC, Resar R, Griffin F, et al. 'Global Trigger Tool' shows that adverse events in hospitals may be ten times greater than previously measured. Health Aff. 2011 Apr; 30(4): 581-88. https: //doi.org/10.1377/hlthaff.2011.0190

[6] Frank J, Brien S. The Safety Competencies Steering Committee. The safety competencies: Enhancing patient safety across the health professions. Canadian Patient Safety Institute, Ottawa, Ontario. 2008.
[7] Smits M, Zegers M, Groenewegen PP, et al. Exploring the causes of adverse events in hospitals and potential prevention strategies. BMJ Qual Saf. 2010 Oct; 19(5): 1-7.

[8] Institute of Medicine. Health professions education: A bridge to quality. National Academies Press, Washington. 2003.

[9] Chenot TM, Daniel LG. Frameworks for patient safety in the nursing curriculum. J Nurs Educ. 2010 Oct; 49(10): 559-68. https : //doi.org/10.3928/01484834-20100730-02

[10] Debourgh GA, Prion SK. Patient safety manifesto: A professional imperative for prelicensure nursing education. J Prof Nurs. 2010 Mar-Apr; 28(2): 110-18. https ://doi .org/10.1016/j .profnu rs.2011.05.001

[11] Mundt MH, Clark MP, Klemczak JW. A task force model for statewide change in nursing education: Building quality and safety. $\mathrm{J}$ Prof Nurs. 2013 Mar-Apr; 29(2): 117-23. https ://doi.org/10.1 016/j.profnurs.2012.12.008

[12] Competencies: June 2010 - May 2015. Canadian Nurses Association; 2013.

[13] Competencies for entry-level Registered Nurse practice. College of Nurses of Ontario; 2014.

[14] Attree M, Cooke H, Wakefield A. Patient safety in an English preregistration nursing curriculum. Nurs Educ Pract. 2008 Jul; 8(4): 239-48.

[15] Mansour M. Current assessment of patient safety education. Br J Nurs. 2008 May; 21(9): 536-43. https://doi.org/10.1016/j. nepr.2007.09.003 
[16] Steven A, Magnusson C, Smith P, et al. Patient safety in nursing education: Contexts, tensions and feeling safe to learn. Nurs Educ Today. 2014 Feb; 34(2): 277-84. https ://doi.org/10.1016/j nedt. 2013.04.025

[17] Maeda S, Kamishiraki E, Starkey J, et al. Patient safety education at Japanese nursing schools: Results of a nationwide survey. BMC Res Notes. 2011 Oct; 17(4): 416-22. https://doi.org/10.1186/17 56-0500-4-416

[18] Howard JN. The missing link: Dedicated patient safety education within top-ranked US nursing school curricula. J Patient Saf. 2010 Sep; 6(3): 165-71. PMid:21491791 https ://doi.org/10.1097/ PTS.0b013e3181eee2ab

[19] Curran JA, Grimshaw JB, Campbell B. Knowledge translation research: The science of moving research into policy and practice. J of Cont Educ Health Prof. 2011 Summer; 31(3): 164-80. https://doi.org/10.1002/chp. 20124

[20] Ginsburg L, Castel E, Tregunno D, et al. The H-PEPSS: An instrument to measure health professionals' perceptions of patient safety competence at entry to practice. BMJ Qual Saf. 2012 Apr; 21(8): 676-84. https://doi.org/10.1136/bmjqs-2011-000601

[21] Duhn L, Karp S, Oni O, et al. Perspectives on patient safety among undergraduate nursing students. J Nurs Educ. 2012 Sep; 15(9): 52631. https://doi.org/10.3928/01484834-20120706-04

[22] Doyle P, Van den Kerkhof EG, Edge DS, et al. Self-reported patient safety competence among Canadian medical students and postgraduate trainees: A cross-sectional study. BMJ Qual Saf. 2015 Nov; 24: 135-41. https://doi.org/10.1136/bmjqs-2014-003142

[23] Borenstein M, Hedges LV, Higgins JPT, et al. Introduction to metaanalysis. John Wiley \& Sons. Hoboken. 2009. https : //doi .org/ 10. $1002 / 9780470743386$

[24] Sullivan GM, Feinn R. Using effect size or why the P value is not enough. J Grad Med Educ. 2012 Sep; 4(3): 279-82. https: //doi.org/10.4300/JGME-D-12-00156.1

[25] Lukewich J, Edge DS, Tranmer J, et al. Undergraduate baccalaureate nursing students' self-reported confidence in learning about patient safety in the classroom and clinical settings: An annual crosssectional study (2010-2013). Int J Nurs Stud. 2015 May; 52(5): 93038. https://doi.org/10.1016/j.ijnurstu.2015.01.010

[26] Graham ID, Logan J, Harrison MB, et al. Lost in knowledge translation: Time for a map? J Cont Educ Health Prof. 2006 Winter; 26(1): 13-24. https://doi.org/10.1002/chp. 47

[27] Barnsley L, Lyon PM, Ralston SJ, et al. Clinical skills in junior medical officers: A comparison of self-reported confidence and observed competence. Med Educ. 2004 Apr; 38(4): 358-67. https : //doi.org/10.1046/j.1365-2923.2004.01773.x

[28] Sulosaari V, Kajander S, Hupli M, et al. Nurse students' medication competence-an integrative review of the associated factors. Nurs Educ Today. 2012 May; 32(4): 399-405. https ://doi .org/10.1 $016 / j$.nedt . 2011.05 .016

[29] Dunn KE. Insight into error hiding: Exploration of nursing student's achievement goal orientations. J Nurs Educ. 2014 Feb; 53(2): 93-6. https://doi.org/10.3928/01484834-20140122-02

[30] Mayo AM, Duncan D. Nurse perceptions of medication errors: What we need to know for patient safety. J Nurs Care Qual. 2004 Sep; 19(3): 209-17. PMid:15326990 https://doi.org/10.1097/00 001786-200407000-00007

[31] Ulanimo VM, O'Leary-Kelley C, Connolly PM. Nurses' perceptions of causes of medication errors and barriers to reporting. $\mathrm{J}$
Nurs Care Qual. 2007 Jan-Mar; 22(1): 28-33. PMid:17149082 https://doi.org/10.1097/00001786-200701000-00007

[32] Bradbury-Jones C, Irvine F, Sambrook S. Empowerment of nursing students in clinical practice: Spheres of influence. J Adv Nurs. 2010 Sep; 66(9): 2061-70. https://doi.org/10.1111/j.1365-264 8.2010.05351.x

[33] Kilcullen NM. Said another way: The impact of mentorship on clinical learning. Nurs Forum. 2007 Apr-Jun; 42(2): 95-104.

[34] Mamchur C, Myrick F. Preceptorship and interpersonal conflict: A multidisciplinary study. J Adv Nurs. 2003 Jul; 43(2): 188-96. https://doi.org/10.1046/j.1365-2648.2003.02693.x

[35] McClure E, Black L. The role of the clinical preceptor: An integrative literature review. J Nurs Educ. 2013 Jun; 52(6): 335-41. https://doi.org/10.3928/01484834-20130430-02

[36] Zilembo M, Monterosso L. Nursing students' perceptions of desirable leadership qualities in nurse preceptors: A descriptive study. Contemp Nurs. 2008 Feb; 27(2): 194-206.

[37] Vaismoradi M. Nursing education curriculum for improving patient safety. Journal of Nursing Education and Practice. 2012 Feb; 2(1): 101-104. http://dx.doi.org/10.5430/jnep.v2n1p101

[38] Barnsteiner J. Teaching the culture of safety. Online J Issues Nurs. 2011 Sep; 16(3): 1-14.

[39] Henneman EA, Roche JP, Fisher DL, et al. Error identification and recovery by student nurses using human patient simulation: Opportunity to improve patient safety. Appl Nurs Res. 2010 Feb; 23(1): 11-21. https://doi.org/10.1016/j.apnr.2008.02.004

[40] Samawi Z, Miller T, Haras MS. Using high-fidelity simulation and concept mapping to cultivate self-confidence in nursing students. Nurs Educ Perspect. 2014 Nov/Dec; 35(6): 408-9. https: //doi.org/10.5480/12-1042.1

[41] Eppich W. "Speaking up" for patient safety in the pediatric emergency department. Clinical Pediatric Emergency Medicine. 2015 Jun; 16(2): 83-9.

[42] Giordana S, Wedin B. Peer mentoring for multiple levels of nursing students. Nurs Educ Perspect. 2010 Nov-Dec; 31(6): 394-6. PMid:21280450

[43] Li HC, Wang LS, Lin YH, et al. The effect of a peer-mentoring strategy on student nurse stress reduction in clinical practice. Int Nurs Rev. 2011 Jun; 58(2): 203-10. https ://doi.org/10.1111/j.14 $66-7657.2010 .00839 . x$

[44] Moscaritolo LM. Interventional strategies to decrease nursing student anxiety in the clinical learning environment. J Nurs Educ. 2009 Jan; 48(1): 17-23. PMid:19227751 https://doi .org/10.3928/0148 4834-20090101-08

[45] Ardalan A, Coppage S, Crouch W. A comparison of student feedback obtained through paper-based and web-based surveys of faculty teaching. British Journal of Educational Technology. 2007 Apr; 38(6): 1085-1101. https://doi .org/10.1111/j.1467-8535.2007.0 0694.x

[46] Dillman DA, Smyth JD, Christian LM. Internet, mail, and mixedmode surveys: The tailored design method (3rd ed.). John Wiley \& Sons, Hoboken. 2009.

[47] Sears K, Godfrey CM, Luctkar-Flude M, et al. Measuring competence in healthcare learners and healthcare professionals by comparing self-assessment with objective structured clinical examinations: A systematic review. JBI Database of Systematic Reviews \& Implementation Reports. 2014 Mar; 12(11): 221-72. https: //doi.org/10.11124/jbisrir-2014-1264 